\title{
Una experiencia de aula centrada en el conocimiento de los dinosaurios
}

\author{
A classroom experience focused on the knowledge of dino-
}

saurs

\author{
MARÍA POUSA \\ Escuela Universitaria CEU de Magisterio de Vigo. \\ MARÍA SANDRA FRAGUEIRO \\ Escuela Universitaria CEU de Magisterio de Vigo.
}

\section{RESUMEN}

En este artículo se presenta un método de enseñanza que aboga por la cooperación y la motivación de los discentes como mecanismo para fomentar el desarrollo de las competencias curriculares en un aula de Educación Infantil. Se fomenta la comunicación, el intercambio de opiniones, la expresión de ideas y sentimientos y la resolución de problemas cercanos a la vida cotidiana del aprendiz, dándole un sentido a lo que aprende desde una perspectiva interdisciplinar y globalizada. Este método se conoce como Trabajo por Proyectos.

Se inicia el presente documento con una fundamentación teórica centrada principalmente en el origen de esta metodología. Posteriormente, se expone el diseño y la implementación de un proyecto basado en "los dinosaurios" en un aula de 4 años en un centro escolar.

\begin{abstract}
In this paper we present a teaching method that advocates cooperation and motivation of students as a mechanism to promote the development of curricular competences in a classroom of Childhood Education. Communication is encouraged, the exchange of opinions the expression of ideas and feelings, and the resolution of problems close to the learner's daily life, giving sense to what they learn from an interdisciplinary and globalized perspective. This method is known as Work by Projects.
\end{abstract}

The present document begins with a theoretical fundamentation mainly centered on the origin of this methodology. Subsequently, we expose the design and implementation of a Project based on "The Dinosaurs" in a 4 years old classroom.

Para citar este artículo: Pousa, M. y Fragueiro, M. S. (2019). Una experiencia de aula centrada en el conocimiento de los dinosaurios EA, Escuela Abierta, 22, 69-89. doi:10.29257/

EA22.2019.07

Recibido: 22/06/2018

Aceptado: 05/04/2019

PALABRAS CLAVES Aprendizaje significativo, dinosaurios, educación infantil, interdisciplinariedad, trabajo por proyectos

\section{KEYWORDS}

Childhood Education,

Dinosaurs, Interdisciplinarity, Significant Learning, Work by Projects. 


\section{INTRODUCCIÓN}

¿Por qué el colegio y el aprendizaje pueden no resultar lo suficientemente atractivos para el alumno? La respuesta a esta pregunta marca uno de los retos que los docentes se plantean en la actualidad, que radica en adecuar, atender y satisfacer las necesidades e intereses de los discentes atendiendo a la confianza, la comprensión, el afecto y el conocimiento de la realidad de forma que les posibilite ser capaces de resolver posibles problemas que se les presenten en la vida.

A lo largo de este documento se pretende mostrar una alternativa de enseñanza a las tradicionales clases magistrales o programaciones basadas en unidades didácticas cerradas. Se busca una metodología más motivadora y cooperativa, en la que el estudiantado sea el protagonista de su propio aprendizaje, dejando el rol del maestro como guía de dicho proceso. El profesor abandona así su función de mero transmisor de conocimientos, para convertirse en creador de espacios y situaciones de aprendizaje beneficiosos para el educando. El papel del maestro es crucial ya que es el encargado de fomentar y crear la motivación, la autoconfianza y la seguridad imprescindibles en el aula.

La educación tradicional puede llegar a ser aislante para el alumnado, incluso puede caer en la monotonía y no generar curiosidad, ni despertar interés ni motivación, es decir, puede resultar poco atractiva para los aprendices. Todavía son muchos los maestros que se mantienen reacios a implementar este tipo de enseñanzaaprendizaje, ya que exige una mayor implicación y esfuerzo, y además requiere un mayor tiempo de dedicación. Es importante conocer los cimientos de este nuevo paradigma en todas sus dimensiones para propiciar correctamente el cambio que supone este marco metodológico.

Como consecuencia de lo expuesto, en este trabajo se plantea como objetivo investigar y profundizar sobre la integración de este enfoque metodológico basado en la programación por proyectos como desafío de una educación de calidad.

Este documento se divide en dos partes. En la primera, se aborda el marco teórico que será la base científica que sustente dicho trabajo, ahondando en la historia, las características y las ventajas que posee dicha metodología. Para finalizar, se desarrolla la propuesta práctica que se diseñó e implementó en un aula de 4 años centrada en "los dinosaurios".

\section{MARCO TEÓRICO}

El aprendizaje basado en proyectos (ABP) surge a finales del siglo XIX y principios del siglo XX sustentado por las raíces del constructivismo (Bruner, 1987; Dewey, 1938; Vygotsky, 1995). En él, el niño aprende realizando construcciones mentales, es decir, construye conocimiento sobre sí mismo y sobre su entorno, teniendo en cuenta sus conocimientos previos, ya que como manifiesta Muñoz (2010) conoce el mundo a través de la interacción con los objetos (físicos y sociales), lo que implica ir comprendiendo sus características y relaciones.

Kilpatrick (1918) estableció las bases del ABP centradas en favorecer el aprendizaje respetando las capacidades innatas del alumnado y enalteciendo el papel activo de los discentes. Influido por Dewey y el movimiento de educación progresista, este precursor defiende la utilización de los intereses del alumnado y la experimentación ya que favorecen el aprendizaje del niño. Su método se basaba en la libertad por el desarrollo natural, en 
el interés y motivación por el trabajo en las experiencias y vivencias, en las necesidades del alumnado y la huida de la enseñanza tradicional.

Este autor plantea una nueva metodología de enseñanza, implantándola en su Escuela Laboratorio de Chicago. En este nuevo enfoque de enseñanza, el aprendizaje es más activo y se apoya en las ideas y experiencias previas del alumno, siendo partícipe en la toma de decisiones.

De la Calle (2016) defiende que Kilpatrick, siguiendo las ideas de Dewey, desarrolló el "método por proyectos" destacando con esta metodología la importancia del alumno que es considerado protagonista, así como también la concepción de la escuela como lugar donde se aborden soluciones a los posibles problemas de los discentes.

Posteriormente, han sido numerosos los estudios realizados sobre esta herramienta educativa impulsando su puesta en práctica y presentándola como una pedagogía emergente (Balongo y Mérida, 2017; Cascales y Carrillo, 2018; Crismán, Cardeñoso y García-González, 2017; Eslava, 2014; Vergara, 2015).

\section{1. ¿Qué es el trabajo por proyectos?}

Cuando nos referimos a proyecto, estamos hablando de un método, de una estrategia metodológica que trata de integrar las necesidades y los intereses del alumnado a las necesidades educativas. Una de sus características principales es la búsqueda de que lo que se aprende y cómo se aprende tengan un sentido para el educando.

Los proyectos son una de las muchas estrategias que puede utilizar el profesorado para organizar todos los contenidos en la escuela desde una perspectiva integradora, donde el aprendizaje crea un clima de comunicación e intercambio de conocimientos. De esta forma, estaremos ayudando al alumno a organizar su pensamiento teniendo muy presente su interés y curiosidad.

Algunas de las concepciones que definen el ABP son las que resaltan que se trata de un procedimiento de aprendizaje que permite alcanzar unos objetivos a través de la puesta en práctica de una serie de acciones, interacción y recursos con el fin de resolver una situación o problema (Díez, 2002). El trabajo por proyectos se caracteriza también por su estructura abierta y flexible, que se va articulando a medida que este se desarrolla considerando que la implicación activa del alumnado es una parte esencial en este proceso (Domínguez, 2013).

Malaguzzi (2001) afirma que lo más importante es que los niños sean capaces de utilizar su capacidad para hacer proyectos y para organizar el trabajo. Este método les permite encontrarse bien dentro de un montón de túneles que tendrán que superar, abandonar y sustituir.

Bravo et al. (2016) indican que, en los procesos de aprendizaje en la etapa infantil, la curiosidad innata es el motor de indagación y el apoyo ofrecido por los docentes les ayuda a ir adquiriendo poco a poco confianza en sus posibilidades para generar saber.

Cascales, Carrillo y Redondo (2017) defienden que el ABP requiere conocer tanto el currículo como los intereses del alumnado, de esta forma garantizamos que el trabajo realizado tenga un buen resultado. Esto conlleva una dedicación y un esfuerzo por parte de maestros y alumnos. 


\section{2. ¿Cómo trabajar por proyectos?}

Vizcaíno (2008) explica que los principios que inspiran el trabajo por proyectos residen en el conjunto de teorías explicativas del aprendizaje: Teoría del aprendizaje social (Vigotsky), Teoría del aprendizaje verbal (Ausubel), y Teoría genética (Piaget), entre otras. Según esta autora, la metodología que debe usar el maestro se inspira en cómo cree que debería ser la educación y para qué debería servir y, por otro lado, también debe tener en cuenta las necesidades del alumno, ya que son los verdaderos protagonistas del aprendizaje.

Es de gran importancia que el profesor tenga en cuenta el momento evolutivo del niño y conocer sus capacidades y destrezas. Debe abordar los contenidos desde una perspectiva globalizadora en la que no se separen por áreas sus conocimientos y experiencias. Es necesario favorecer que el aprendizaje del niño sea significativo, de forma que el alumno relacione el nuevo aprendizaje con experiencias propias. El juego se contempla como un instrumento privilegiado para el desarrollo de las capacidades del educando, además de ser un gran agente motivador. Hay que tener en cuenta también que el escolar aprenda mientras interactúa con sus iguales, favoreciendo así el desarrollo de capacidades afectivas y sociales, pero también es importante tener en cuenta la atención individualizada, pues cada niño tiene unas motivaciones y unas capacidades diferentes. Se debe procurar crear un clima de seguridad y confianza donde puedan surgir vínculos afectivos. Por último, contar con la familia, dado que es el contexto educativo más importante para el alumno.

Por otra parte, Domínguez (2013) expone que la metodología que se puede emplear en el aula se puede calificar como metodología de investigación. Cuando se habla de investigar en Educación Infantil, se refiere al proceso de buscar soluciones a problemas que surgen día a día en los niños y que forma parte de sus intereses. Por otra parte, cuando se habla de investigación en el aula, la pregunta o problema puede surgir de un alumno, del grupo o incluso del profesor. Tiene como punto de partida la existencia de un problema que crea curiosidad e interés en el colegial. También es importante destacar que tanto la observación como la exploración son las características básicas de cualquier proceso de investigación.

Estas dos propuestas de metodología, aun siendo diferentes, tienen como eje central al alumno como protagonista del aprendizaje, por lo que cumplen a la perfección con las características del trabajo por proyectos.

Para implementar un proyecto es necesario seguir una estructura secuenciada en el aula. Vizcaíno (2008) marca los siguientes pasos:

\section{$1^{\circ}$ Elección del Proyecto}

La elección del proyecto parte de los intereses del niño, de la clase como grupo. Cuando se empieza a trabajar con esta metodología es importante que el maestro se sienta seguro, por tanto, los primeros proyectos pueden surgir del profesor. Es primordial que se sienta a gusto y conozca y domine el tema. Si el tema lo inicia el educador es conveniente aportar una fuerte motivación inicial.

Un proyecto surge de la necesidad de dar respuesta a una cuestión la cual da sentido a una investigación. Sobre ese tema se organiza el trabajo de modo que todas las áreas de currículo quedan globalizadas y dotadas de significatividad (Aragón, 2017). 


\section{$2^{\circ}$ Reconocimiento de ideas previas}

Una de las características más importantes del trabajo por proyectos es que favorece el aprendizaje significativo. Por lo tanto, es importante conocer cuáles son los conocimientos previos del alumno y, a partir de ellos, analizar y aclarar las dudas. Es por esto por lo que la asamblea se organiza en torno al “¿Qué sabemos?”.

\section{3o Planificación y desarrollo de las ideas}

Se debe observar qué es lo que le interesa a los alumnos, es decir, sobre qué les gustaría aprender. A través de la asamblea se analizan, escuchan y anotan todos los interrogantes y aportaciones de los aprendices. Con toda esta información se establece “¿Qué queremos saber?".

\section{$4^{0}$ Organización y propuestas de actividades}

Aquí es importante tener en cuenta los intereses de los alumnos. Se deben incluir actividades propuestas por el alumnado. Al mismo tiempo, el docente también puede aportar las actividades que crea oportunas y necesarias para la marcha del proyecto.

\section{$5^{\circ}$ Búsqueda de información}

Internet es una fuente con la que fácilmente se puede encontrar información sobre cualquier temática, por lo tanto, es conveniente emplear todo tipo de textos (periódicos, revistas, libros de consulta, etc.).

Muñoz (2010) afirma que es muy importante el papel que se le otorga al alumno a la hora de recopilar información, puesto que se busca que formulen preguntas, busquen respuestas, las pongan en común y debatan sobre ellas.

Es sustancial informar a las familias sobre lo que se está realizando en clase para que aporten todo el material necesario. Gran parte del éxito de un proyecto reside en la buena comunicación, implicación y colaboración con las familias.

\section{$6^{0}$ Recopilación, organización y estudio de la información. Materiales y recursos obtenidos}

El tiempo destinado a la asamblea es fundamental para observar todos los materiales aportados, comentarlos y resolver posibles dudas. Ese será uno de los momentos principales y especiales, ya que es aquí donde verdaderamente se desarrolla el proyecto. Por ello, se le dedicará todo el tiempo necesario para que los niños interpreten, analicen, piensen y construyan su aprendizaje.

\section{Elaboración de las actividades}

Es esencial que se abarquen las tres áreas de contenidos en las actividades propuestas y que sean de todo tipo: grupales, individuales, salidas, colaboraciones con familias, etc.; sobre todo, que favorezcan la creatividad y la investigación. Un elemento importante para motivar a los alumnos es la decoración de la clase. 


\section{8o Sintesis y evaluación}

Una forma original y motivadora para finalizar el proyecto es crear algo especial como, por ejemplo, realizar una merienda, una excursión, una foto, etc.

En cuanto a la evaluación, como en cualquier metodología, el trabajo por proyectos también debe ser evaluado. Para comprobar si se lograron o no los objetivos propuestos, se puede utilizar la observación participante y sistemática que ayuda en la toma de decisiones facilitando el cambio si fuese necesario.

\subsection{Desarrollo de las competencias en el trabajo por proyectos.}

La referencia legislativa en la que se basa este trabajo y en la que se apoya es el Decreto 330/2009, de 4 de junio, por el que se establece el currículo en la Comunidad Autónoma de Galicia que permanece vigente en la actualidad. "La Ley orgánica 2/2006, de 3 de mayo, de educación, en su artículo 6, define el currículo como el conjunto de objetivos, competencias básicas, contenidos, métodos pedagógicos y criterios de evaluación" (Decreto 330/2009, p. 10.773). Se entiende por competencia a la capacidad del niño de poner en práctica, en contextos y situaciones diferentes, conocimientos teóricos, habilidades, conocimientos prácticos y actitudes. El concepto de competencia va más allá del saber hacer o aplicar y del saber estar. El ABP contribuye a desarrollar las competencias curriculares (Arias et. al., 2009):

\section{- Autonomía e iniciativa personal:}

Cuando se trabaja a través del ABP, los alumnos desarrollan la creatividad, la capacidad de tomar decisiones, aportar ideas y respetar las de los demás, adquieren responsabilidades en el proceso de enseñanza-aprendizaje y toman iniciativa en la resolución de problemas.

\section{- Competencia para aprender a aprender:}

Una de las características principales del trabajo por proyectos es que es el propio alumno el que decide sobre qué quiere aprender. Son ellos los que plantean preguntas y buscan información para resolver posibles problemas.

Con el ABP se promueve la participación y motivación del alumnado, realizando actividades cooperativas, manipulativas y de observación.

\section{- Competencia en comunicación lingüística:}

A través de este método los alumnos aprenden a expresar necesidades, sentimientos e ideas, a respetar las normas establecidas, aprenden a escuchar y comprender las aportaciones de los demás. Es a través de la comunicación entre los niños cuando desarrollan de una mejor forma la construcción de frases.

\section{- Competencia matemática:}

Mediante el ABP se puede crear alguna actividad en la que el aprendiz tenga que razonar para llegar a una posible solución. También se pueden plantear actividades en las que se realicen ejercicios de conteo, de sumas o restas a través de la manipulación de objetos, de seriaciones, etc. 


\section{- Tratamiento de la información y competencia digital:}

El trabajo por proyectos acerca a los alumnos a los diferentes aparatos tecnológicos a través de diferentes actividades como, por ejemplo, la búsqueda de información, la visualización de algún vídeo en Internet, la realización de ejercicios en la pantalla digital, la utilización de tablet, etc.

- Competencia en el conocimiento y la interacción con el mundo físico:

A través de esta metodología los alumnos pueden realizar observaciones y explorar el mundo que los rodea, mediante actividades relacionadas con la naturaleza los alumnos desarrollan la capacidad de responsabilidad, preocupación y cuidado por el medio ambiente.

\section{- Competencia social y ciudadana:}

Gran parte de las actividades realizadas en el ABP se realizan en grupo, favoreciendo la comunicación y el respeto entre los alumnos. Gracias a esto, se crean situaciones de diálogo e intercambio de opiniones en las que el niño debe escuchar y respetar otras ideas.

\section{- Competencia cultural y artística:}

En el trabajo por proyectos se realizan multitud de actividades en las que se requiere la creatividad por parte del niño, como por ejemplo, actividades plásticas en las que el niño emplea todo tipo de materiales, despertando así su lado más artístico. Utilizando los proyectos podemos trabajar de diferentes formas, a través de la música, de los juegos, de danzas, etc.

\subsection{Impacto de esta metodología en las aulas}

La metodología por proyectos permite establecer una relación distinta entre el conocimiento y el aprendizaje. Permite abordar las necesidades de los alumnos de un modo diferente, contribuyendo a desarrollar competencias para la vida.

Hay que tener en cuenta que la aplicación del ABP puede presentar dificultades. Un cambio metodológico implica en buena medida un paso hacia algo desconocido y, a veces, ello conlleva el temor de que se nos vaya de las manos (De la Calle, 2016). Los alumnos pueden cambiar con rapidez sus gustos y no es seguro que se alcancen todas las metas propuestas por falta de tiempo (Erre, 2011; Calleja, 2014). Por el contrario, son numerosos los beneficios que presenta esta metodología: aumenta la motivación y la participación del alumnado; a través de actividades colaborativas los niños aprenden a trabajar en equipo, a comunicarse, a tomar decisiones, etc.; integra al aprendizaje aspectos de la realidad del niño; se desarrolla la habilidad de resolución de problemas; el alumno recopila información necesaria para solventar dudas... (Santos y Gómez, 2012). 


\section{PROPUESTA DE INTERVENCIÓN}

Como forma de aproximarse al trabajo por proyectos $(\mathrm{ABP})$ se presenta una colección de actividades con un centro de interés común (los dinosaurios) susceptible de ser reorganizadas y con potencial de constituir un proyecto para trabajar en Ed. Infantil. Se puso en práctica en un CEIP situado en un municipio de la provincia de Pontevedra. En cuanto a la situación geográfica, el centro está situado en una zona costera. La población está muy dispersa lo que dificulta la socialización de los niños. Es un centro que cuenta con una línea para los tres cursos de Educación Infantil y una línea para cada curso de Educación Primaria. Se llevó a cabo con alumnos de $5^{\circ}$ curso de Educación Infantil. El grupo estaba formado por 23 alumnos de los cuales 14 son niñas y 9 son niños.

Atendiendo a las diferentes necesidades del alumnado y, por supuesto, al interés mostrado por el tema, el proyecto se realizó a lo largo de un mes, al que se le dedicaron dos horas diarias.

Para la realización de las actividades se empleó fundamentalmente el aula en su totalidad, se unieron las mesas para realizar las actividades plásticas, se empleó la asamblea para contar cuentos, realizar lluvia de ideas y el rincón de los dinosaurios. Además de este espacio, también se usaron la biblioteca y la sala de informática para llevar a cabo la búsqueda de información. Por otra parte, se utilizó el patio para desarrollar determinadas actividades.

\subsection{Objetivos}

El objetivo principal que se planteó al implementar este ABP fue obtener conocimientos sobre la evolución, la alimentación, la reproducción, la desaparición de los dinosaurios; así como de algunas de las especies existentes, realizando diversas actividades plásticas y de investigación individuales y grupales encaminadas a la consecución de un evento como producto final de dicho proyecto.

\subsection{Metodología}

Todas las actividades realizadas fueron lúdicas y teniendo muy en cuenta las experiencias previas de los educandos. Por lo tanto, el aprendizaje fue, principalmente, significativo y a través del juego. De esta manera, se incluyeron los siguientes principios metodológicos:

- Principio de juego.

- Construcción de aprendizajes significativo.

- Principio de actividad.

- Creación de un ambiente cómodo para el alumnado y, por supuesto, establecer una relación de confianza con el profesor.

- Principio de socialización. 


\subsection{Actividades}

\subsection{7. ¿Cómo surgió el proyecto?}

El proyecto surgió a raíz del interés de uno de los alumnos por el mundo de los dinosaurios. Una mañana trajo a la asamblea un dinosaurio de peluche y contó de qué especie era, qué comía, dónde vivía, etc. Al percibir el gran interés que despertó y a raíz de los interrogantes que formularon sus compañeros en el aula se decidió iniciar el proyecto centrado en el mundo de los dinosaurios.

La resolución de estos interrogantes se realizó a partir de la reflexión y la participación de los alumnos, por lo tanto, el aprendizaje fue significativo.

\subsubsection{Fase de preparación}

Se inició el proyecto con una actividad de introducción-motivación: huellas de dinosaurio. Se prepararon una serie de huellas de dinosaurio, empleando cartulina, que se pegaron por el aula antes de que los alumnos llegasen. Por otra parte, se elaboró un huevo de dinosaurio y en su interior se introdujo un dinosaurio con una nota como se muestra en la Figura 1.

\section{Figura 1}

Huevo de dinosaurio y nota informativa

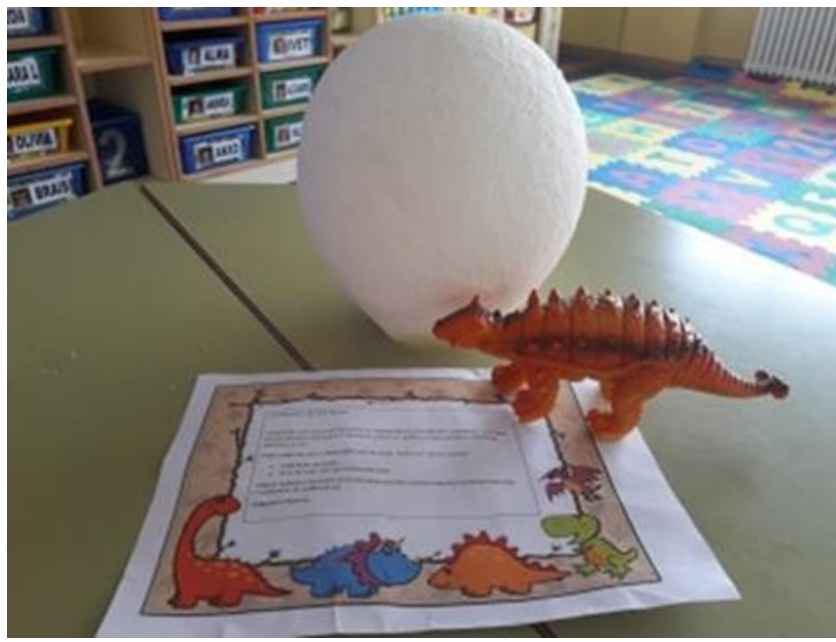

Cuando los alumnos entraron en el aula se encontraron por sorpresa la clase ambientada, como puede observarse en la Figura 2. Debían decir de qué podían ser esas huellas y seguirlas hasta encontrar el huevo. Una vez hallado, el encargado lo abrió y mostró lo que contenía. Posteriormente, una de las profesoras leyó la nota. 


\section{Figura 2}

Huellas de dinosaurio

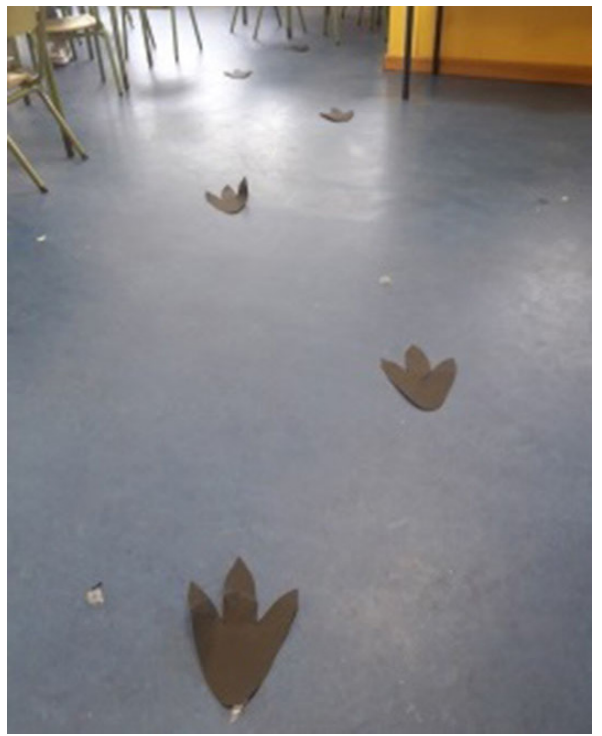

El dinosaurio fue la mascota durante todo el proyecto. En grupo y mediante una votación, los alumnos escogieron el nombre.

\subsection{3. ¿Qué sabemos? ¿Qué queremos saber?}

Mediante una puesta en común y el registro de las concepciones, ideas, creencias... los alumnos tuvieron que pensar, expresarse y comunicarse. Esto lo realizaron mediante una lluvia de ideas y una serie de preguntas pautadas por el maestro.

Con toda esta información se confeccionó un mural en el que se anotaron todas las preconcepciones que los alumnos aportaron, tal y como se muestra en la Figura 3.

Se configuró un guion que permitió establecer los objetivos a alcanzar y los contenidos a desarrollar, es decir, un índice que permitió ir organizando la información. Se realizó una lista de curiosidades formuladas en preguntas que les surgieron a los niños a lo largo de este proceso, tal y como se observa en la Figura 4. 


\section{Figura 3}

Mural “Qué sabemos?”

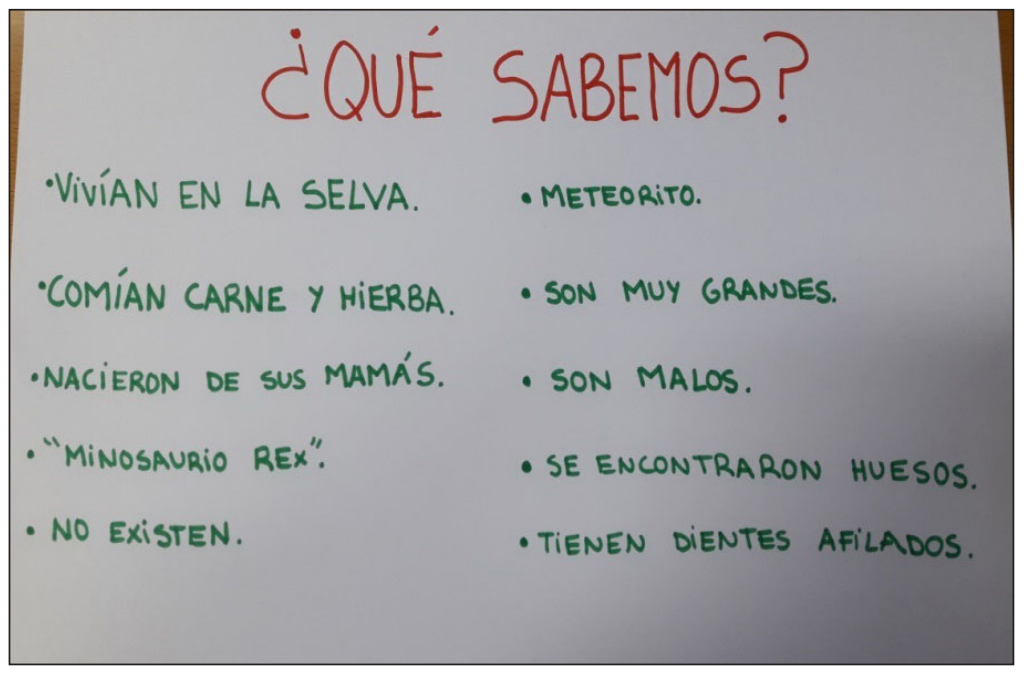

Figura 4

Mural “Qué queremos saber?”

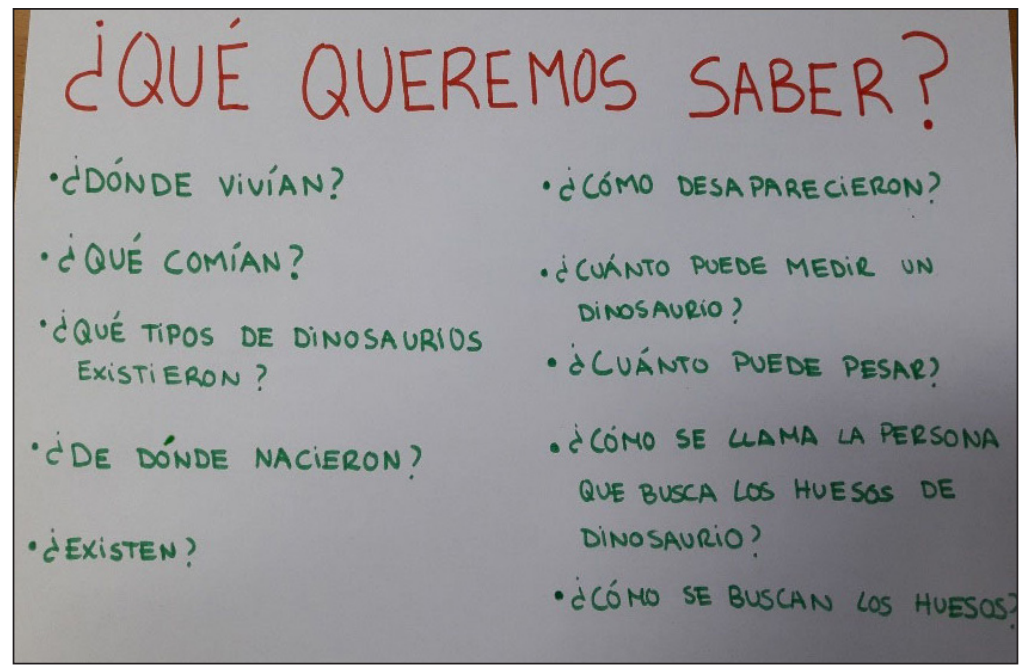

\subsubsection{Fase de desarrollo}

En este apartado, se detallan algunas de las actividades de desarrollo realizadas partiendo de la información buscada, aportada por las familias y en el propio centro. 


\section{- Rincón de los dinosaurios}

Para la elaboración de nuestro rincón se pidió la colaboración de las familias a través de una nota informativa, en la que se les comunicó que podían aportar cualquier objeto, libro, muñeco, cuento, etc., relacionado con el tema. Además, se realizó la búsqueda de información en la biblioteca y en la sala de informática del Centro.

Con todo el material aportado, se elaboró el rincón de los dinosaurios al que los alumnos podían acudir siempre que necesitasen buscar información, observar, jugar, etc.

\section{- Huevosaurios}

Para elaborar cada huevo se empleó una pasta formada por harina, agua y café. Con las manos se dio forma de huevo a la mezcla dejando en el interior un pequeño dinosaurio en cada uno de ellos. Una vez acabados se introdujeron en el horno durante 35 minutos a $130^{\circ}$. De esta forma, se pudieron trabajar las medidas, las cantidades, el tiempo y la motricidad fina.

\section{- Alimentación de los dinosaurios}

Se elaboró un mural que estaba dividido en parte carnívora y parte herbívora. En la parte herbívora se dibujaron dinosaurios que consumían vegetales y en la parte carnívora, dinosaurios que consumían carne. En ambas fracciones se dibujó un carrito de la compra. Con ayuda de recortes de revista se llenaron ambos carritos de la compra de los dinosaurios, clasificando alimentos de origen animal y alimentos de origen vegetal.

- Taller: Somos paleontólogos

Para esta actividad se desplazaron al patio. Se precintó el arenero con cinta amarilla simulando el espacio de trabajo de los paleontólogos. Se repartieron a los discentes objetos de trabajo como palas, rastrillos o pinceles para que buscasen los huesos de dinosaurios escondidos en el arenero. Estos huesos se guardaron para realizar la siguiente actividad.

\section{- Esqueleto de dinosaurio}

Se repartió a los alumnos un cartón sobre el que pegaron un conjunto de huesos para formar el esqueleto de un dinosaurio. En el cartón estaba dibujada la silueta del dinosaurio para que fuese más sencillo construirlo.

$$
\text { - Los fósiles }
$$

Para realizar esta actividad únicamente se necesitaron arcilla para modelar y dinosaurios de juguete. Los alumnos amasaron la arcilla hasta que quedó blanda y moldeable, hicieron bolitas y las aplastaron. Finalmente, escogieron los dinosaurios que más les gustaban para imprimirlos en la masa y los dejaron secar durante dos días.

\section{- ¿Cuánto mide la huella de un dinosaurio?}

Con ayuda de una cartulina en forma de huella de dinosaurio, a tamaño real, se hizo comprender a los niños la gran diferencia de tamaño existente entre sus huellas y las de los dinosaurios. Se colocaron los zapatos de los alumnos en la huella para interiorizar las dimensiones.

\section{- Comparamos nuestras huellas}

Esta actividad se centró en la realización de una ficha didáctica en la que aparecía la huella de un dinosaurio y, al lado, un espacio en blanco en el que tuvieron que dejar su huella de la mano impresa con témpera. Así, pudieron ser conscientes de la diferencia de forma entre unas y otras huellas. En la Figura 5 se muestra una de las fichas realizadas por uno de los alumnos. 


\section{Figura 5}

Ficha didáctica sobre la comparación de huellas"

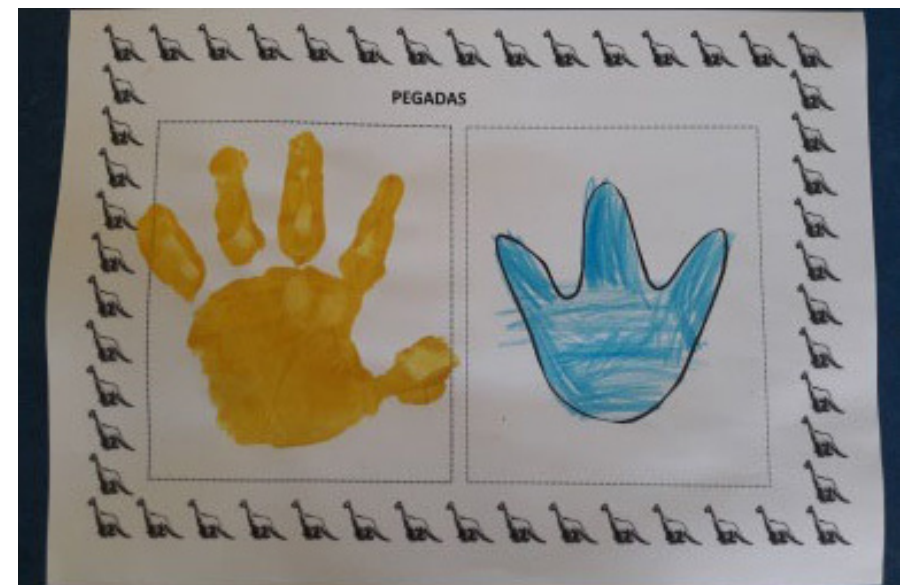

- Dinonúmeros

Se realizaron diez tarjetas en forma de dinosaurio, las cuales tenían un número situado en la barriga. Este juego consistió en que los alumnos colocasen tantas pinzas como indicaba el número del dinosaurio en su cuerpo. Estas tarjetas se pueden observar en la Figura 6.

\section{Figura 6}

\section{Tarjetas Dinonúmeros}

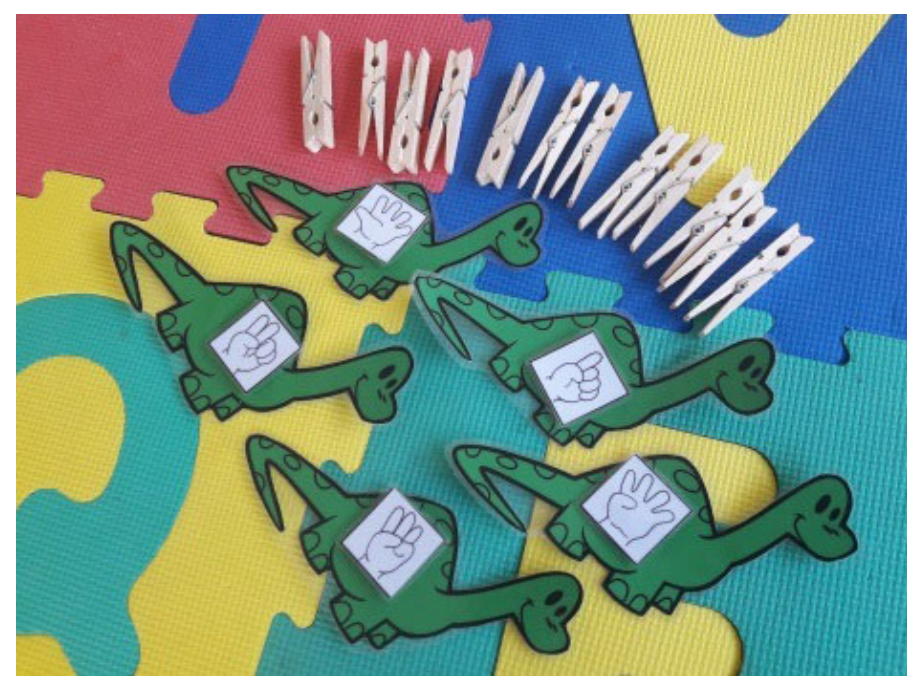




\section{- Partes de un dinosaurio}

Se realizó un mural con los dinosaurios más destacados y se indicaron las partes de cada uno con flechas. Los alumnos, por grupos, decoraron y pintaron el mural a su gusto. Una vez finalizado, ellos mismos escribieron los nombres de las partes de cada dinosaurio. Se puede observar en la Figura 7 cómo los alumnos realizaron esta actividad. Por otra parte, en la Figura 8, se muestra el mural trabajado en el aula ya finalizado.

\section{Figura 7}

Alumnos elaborando el mural de los dinosaurios

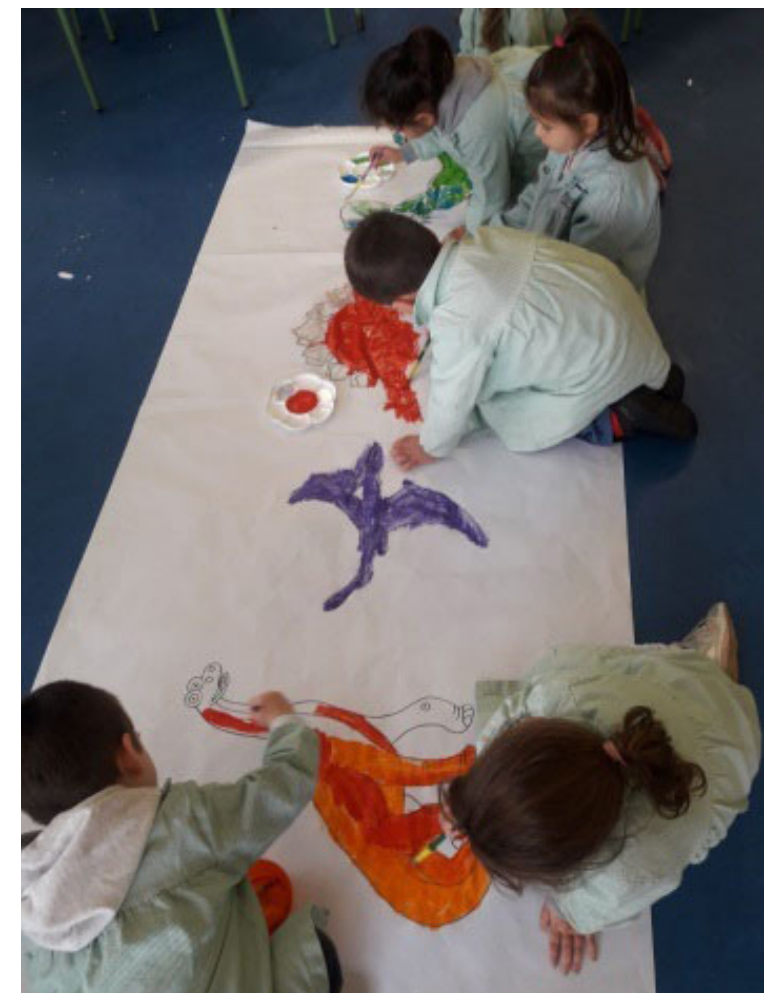




\section{Figura 8}

Mural de los dinosaurios

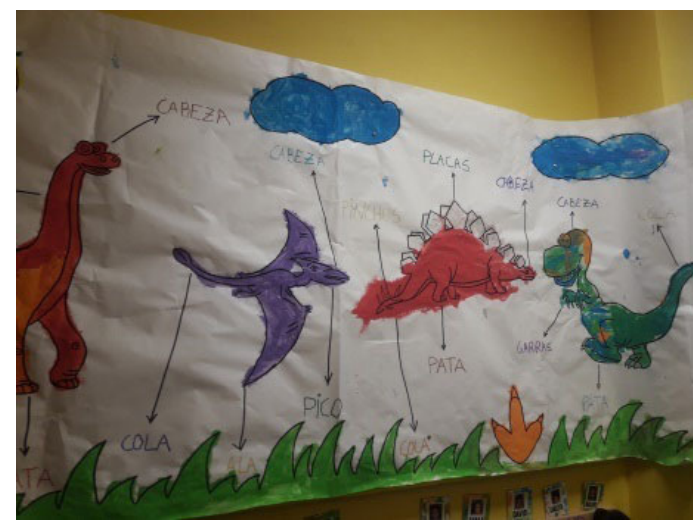

- Caza de dinosaurios

La actividad consistió en buscar los códigos QR distribuidos previamente por la planta baja del Centro. Cada código contenía la imagen de una especie de dinosaurio diferente sobre un fondo blanco para que los alumnos centrasen su atención sobre dicha imagen. Finalmente, en la asamblea manifestaron en común de qué dinosaurio se trataba, cómo era, qué comía, etc. En la Figura 9 se puede observar un ejemplo de dicha actividad.

\section{Figura 9}

\section{Cazando códigos QR}

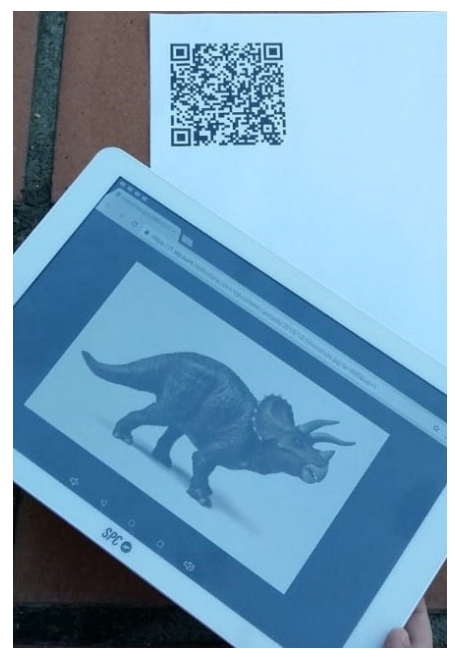

- Huevo de dinosaurio

Se consiguió un huevo de dinosaurio para esta actividad, el cual se muestra en la Figura 10. Este huevo contenía un dinosaurio de goma en su interior y para que eclosionase fue necesario introducirlo en un recipiente con agua y dejarlo sumergido en esta durante una semana. En la asamblea se comentó la diferencia del huevo con el paso de los días hasta su eclosión. 
Figura 10

Alumnos observando el huevo de dinosaurio

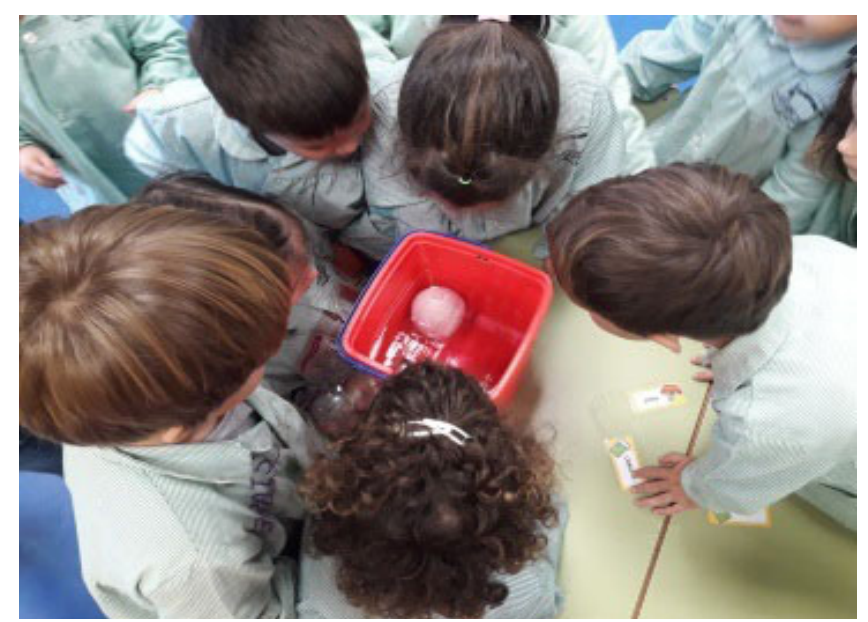

\subsubsection{Fase final}

Se finalizó el proyecto con una actividad (producto final) que consistió en la puesta en práctica de un evento como colofón. Consistió en la exposición oral, en la que los aprendices mostraron los trabajos realizados y explicaron lo que trabajaron a los otros grupos invitados de Educación Infantil y Primaria del Centro. Esta actividad sirvió para dignificar el fruto de todo el trabajo realizado a lo largo de las distintas sesiones dedicadas a este proyecto.

\subsection{Evaluación}

Para conocer el grado de aprendizaje de los alumnos y comprobar si alcanzaron o no los objetivos previstos, se utilizó la observación participante y sistemática. Se analizó si el alumno estaba motivado, su interés, el grado de participación, etc.

La evaluación constó de tres partes. Primeramente, se realizó una evaluación inicial para conocer cuál era el punto de partida de los alumnos. Por otra parte, se evaluó el desarrollo del proyecto, donde se realizó un seguimiento de la evolución de los discentes, principalmente a partir de la observación. Y, para concluir, se llevó a cabo una evaluación final a través de la recogida de los conocimientos finales de los niños, rellenando una rúbrica de evaluación cuyos resultados se muestran en la Tabla 1. 
Tabla 1.

Resultados de la evaluación

\section{RESULTADOS EVALUACIÓN}

SIEMPRE

A VECES

NUNCA

EVALUACIÓN INICIAL

\begin{tabular}{|c|c|c|c|}
\hline Participa activamente en la elección del proyecto & $95,66 \%$ & $4,34 \%$ & $0 \%$ \\
\hline Escucha y respeta las ideas & $91,30 \%$ & $8,70 \%$ & $0 \%$ \\
\hline Se muestra motivado & $86,95 \%$ & $13,05 \%$ & $0 \%$ \\
\hline \multicolumn{4}{|c|}{ DESARROLLO } \\
\hline Aporta ideas para realizar & $95,66 \%$ & $4,34 \%$ & $0 \%$ \\
\hline Busca información & $95,66 \%$ & $4,34 \%$ & $0 \%$ \\
\hline Participa en las actividades & $100 \%$ & $0 \%$ & $0 \%$ \\
\hline Continúa motivado & $100 \%$ & $0 \%$ & $0 \%$ \\
\hline Trabaja de forma cooperativa & $100 \%$ & $0 \%$ & $0 \%$ \\
\hline Conoce el concepto "herbívoro" & $100 \%$ & $0 \%$ & $0 \%$ \\
\hline Conoce el concepto "carnívoro" & $100 \%$ & $0 \%$ & $0 \%$ \\
\hline Descubre qué es un fósil y cómo se forma & $100 \%$ & $0 \%$ & $0 \%$ \\
\hline Identifica palabras específicas sobre el tema & $95,66 \%$ & $4,34 \%$ & $0 \%$ \\
\hline Asocia los números del 1 al 10 con sus cantidades correspondientes & $95,66 \%$ & $4,34 \%$ & $0 \%$ \\
\hline \multicolumn{4}{|c|}{ FINAL } \\
\hline Tiene conocimiento sobre el tema & $100 \%$ & $0 \%$ & $0 \%$ \\
\hline Diferencia dinosaurios herbívoros de carnívoros & $100 \%$ & $0 \%$ & $0 \%$ \\
\hline Conoce las especies de dinosaurios trabajadas & $95,66 \%$ & $4,34 \%$ & $0 \%$ \\
\hline Conoce la profesión del paleontólogo & $100 \%$ & $0 \%$ & $0 \%$ \\
\hline Cuenta de forma sencilla cómo desaparecieron los dinosaurios & $95,66 \%$ & $4,34 \%$ & $0 \%$ \\
\hline Ha mejorado estrategias de participación & $100 \%$ & $0 \%$ & $0 \%$ \\
\hline Expresa aquellos conocimientos que le han resultado significativos & $100 \%$ & $0 \%$ & $0 \%$ \\
\hline
\end{tabular}

Fuente: elaboración propia

El desarrollo del proceso de enseñanza-aprendizaje también debe ser evaluado para saber si se alcanzaron con éxito los objetivos propuestos. Para ello se debe analizar tanto el proyecto como la actuación del docente que fueron evaluados utilizando la rúbrica cuyos resultados se muestran en la Tabla 2. A partir de las respuestas obtenidas en dicha rúbrica se extrajo una reflexión con la que concluiremos este artículo. 
Tabla 2.

Resultados de la autoevaluación del docente

\begin{tabular}{|c|c|c|c|}
\hline & BIEN & REGULAR & MAL \\
\hline Investigo con mis alumnos & $\mathrm{X}$ & & \\
\hline Desarrollo el pensamiento crítico de los alumnos & $\mathrm{X}$ & & \\
\hline Genero centros de interés y motivación & $\mathrm{X}$ & & \\
\hline Propongo actividades motivadoras y lúdicas & $\mathrm{X}$ & & \\
\hline Favorezco la autonomía de los alumnos & $\mathrm{X}$ & & \\
\hline Implico a los alumnos en situaciones de diálogo y reflexión & $\mathrm{X}$ & & \\
\hline Respeto los ritmos de aprendizaje de los alumnos & $\mathrm{X}$ & & \\
\hline Fomento un aprendizaje significativo y globalizador & $\mathrm{X}$ & & \\
\hline Trabajo en función de la planificación & & $\mathrm{X}$ & \\
\hline Propongo actividades cooperativas & $\mathrm{X}$ & & \\
\hline Adecúo los recursos materiales & $\mathrm{X}$ & & \\
\hline Utilizo los recursos tecnológicos & $\mathrm{X}$ & & \\
\hline Me apoyo en materiales de editoriales comerciales & & $\mathrm{X}$ & \\
\hline Conozco las bases que sustentan el ABP & $\mathrm{X}$ & & \\
\hline
\end{tabular}

Fuente: elaboración propia

\section{CONCLUSIÓN}

Como conclusión al trabajo realizado en el CEIP, tras analizar los resultados obtenidos en la evaluación que se pueden visualizar en la Tabla 1, al inicio del proyecto tres alumnos presentaban falta de atención y escasa motivación. A medida que se avanzó en la realización de las diferentes actividades, estos mostraron una cierta evolución, pues se consiguió generar en ellos la necesidad de aprender y poner en práctica los conocimientos adquiridos para llevar a cabo el evento final delante del resto de compañeros.

Todos los alumnos han alcanzado los objetivos programados, aunque un alumno presentó una mayor dificultad en la adquisición de algunos aprendizajes ya que poseía problemas en la comprensión del lenguaje, lo que se fue mejorando con una mayor atención individualizada para la realización de las tareas. Fueron muchos los puntos ligados a una autopercepción positiva como, por ejemplo, la alta motivación del alumnado, la expresión de ideas propias, el desarrollo de capacidades a la hora de buscar información, el trabajo colaborativo, el fomento de la creatividad y el desarrollo del pensamiento crítico de los discentes entre otros. 
Cabe destacar que se mejoraron los resultados académicos así como la motivación y el clima en el aula de los alumnos, lo que concuerda con estudios publicados, como por ejemplo, el de Vega (2015) y Sánchez (2013).

Gracias a la implementación del trabajo, como se puede extraer de los resultados de la autoevaluación docente (Tabla 2), se desarrollaron competencias como promover y facilitar los aprendizajes en Educación Infantil desde una perspectiva globalizadora, diseñar y regular espacios de aprendizaje, fomentar la convivencia en el aula y abordar la resolución de conflictos, reflexionar sobre las prácticas del aula para innovar y mejorar la labor docente, desarrollar la capacidad de organización y planificación y, por último, relacionar la teoría y práctica con la realidad del aula.

"La admirable capacidad de sorpresa de los más pequeños, el asombro y el interés por conocer todo lo que les rodea, nos permite y obliga a la vez al profesorado a embarcarnos en proyectos cuyos derroteros siguen caminos inesperados, cualidad que comparten con su origen: la sorpresa” (Bravo et al., 2016, p. 135).

\section{REFERENCIAS}

Aragón, A. (2017). Aprendizaje basado en proyectos: ventajas e inconvenientes de su puesta en marcha en educación infantil. La Rioja: Servicio de Publicaciones.

Arias, A., Arias, D., Navaez, M. y Rial, M. (2009). O traballo por proxectos en infantil, primaria e secundaria. Galicia: Xunta de Galicia.

Balongo, E. y Mérida, R. (2017). Proyectos de trabajo: una metodología inclusiva en Educación Infantil. Revista Electrónica de Investigación Educativa, 19(2), 125-142. doi: https://doi.org/10.24320/redie.2017.19.2.1091

Bravo, M., Corpas, B., Encinas, M., González, M., Guzmán, O., Lara, M., León, M., López, M., Mérida, R., Olivares, M. y Sánchez, N. (2016). Los Proyectos de Trabajo. Tejiendo sueños, construyendo vida en la escuela infantil. Madrid: Ediciones Pirámide.

Bruner, J. S. (1987). La importancia de la educación. Barcelona: Paidós.

Calleja, J. (2014). Ventajas y desventajas del aprendizaje basado en proyectos. Recuperado de http://mitarima.jgcalleja. es/2014/04/08/ventajas-y-desventajas-del-aprendizaje-basado-en-proyectos/

Cascales, A., Carrillo, M. E. y Redondo, A. (2017). ABP y tecnología en Educación Infantil. Revista de Medios y Educación, 50, 201-210. doi: http://www.redalyc.org/articulo.oa?id=36849882014

Cascales, A. y Carrillo, M. E. (2018). Aprendizaje basado en proyectos en educación infantil: cambio pedagógico y social. Revista Iberoamericana de Educación, 76, 79-98. Recuperado de https://rieoei.org/RIE/article/view/

Crismán, R., Cardeñoso, J. M. y García-González, E. (2017). La evaluación del trabajo por proyectos para Educación infantil: un estudio con estudiantes universitarios a partir de un proyecto de innovación docente. Revista de Docencia Universitaria, 15(2), 35-55. doi: https://doi.org/10.4995/redu.2017.5956

Decreto 330/2009, de 4 de junio, por el que se establece el currículo de la educación infantil en la Comunidad Autónoma de Galicia. Diario Oficial de Galicia. Galicia, 23 de junio de 2009, núm. 121, pp. 10773-10799. 
De la Calle, M. (2016). Aprendizaje basado en proyectos (ABP): posibilidades y perspectivas en ciencias sociales. Íber. Didáctica de las Ciencias Sociales, Geografía e Historia, 82, 7-12.

Dewey, J. (1938). Experience and Education. New York: Touchstone.

Díez, C. (2002). La oreja verde de la escuela. Trabajo por proyectos y vida cotidiana en la escuela infantil. Madrid: Ediciones de la Torre.

Domínguez, G. (2013). Proyectos de trabajo. Una escuela diferente. Madrid: La Muralla.

Erre, E. (2011). Enriquécete y enriquécelos: trabaja por proyectos. Recuperado de http://avolarpajarillo.blogspot.com. es/2011/04/grriyug-qerylr3.html

Eslava, C. (2014). Ambientes para la infancia: escuelas entre experiencia y proyecto. Revista Latinoamericana de Educación Infantil, 3(2), 51-80. Recuperado de http://www.reladei.net

Kilpatrick, W. H. (1918). The Project Method: The Use of the Purposeful Act in the Educative Process. Recuperado de https://archive.org/details/projectmethodus00kilpgoog

Malaguzzi, L. (2001). La Educación Infantil en Regio Emilia. Barcelona: Octaedro.

Muñoz, M. (2010). El trabajo por proyectos en Educación Infantil. Recuperado de http://www.actiweb.es/dg9/archivo7.pdf

Sánchez, J. (2013). Qué dicen los estudios sobre el aprendizaje basado en proyectos. Recuperado de http://www.actualidadpedagogica.com/estudios_abp

Santos, A. y Gómez, B. (2012). Competencias para la Inserción Laboral. Guía para el Profesorado. Madrid: Ministerio de Educación, Cultura y Deporte. Recuperado de http://www.sepe.es/LegislativaWeb/verFichero. do?fichero=09017edb800f 8507

Vega, V. (2015). Progect-Based Learning Reserch Review. Recuperado de http://www.edutopia.org/pbl-researchlearning-outcomes

Vergara, J. J. (2015). Aprendo porque quiero. El Aprendizaje Basado en Proyectos (ABN), paso a paso. Madrid: SM.

Vizcaíno, M. (2008). Guía fácil para programar en Educación Infantil (0-6 años). Madrid: Wolters Kluwer.

Vygotsky, L. S. (1995). Pensamiento y Lenguaje. Barcelona: Paidós.

\section{INFORMACIÓN SOBRE LAS AUTORAS}

María Sandra Fragueiro Barreiro. Doctora en Química Analítica por la Universidad de Vigo (2004) y Premio Extraordinario de Doctorado (Universidad de Vigo, 2006). Actualmente ejerce como Profesora Acreditada en la Escuela Universitaria CEU de Magisterio de Vigo en el Departamento de Ciencias. Es Coordinadora del Trabajo Fin de Grado en dicha Escuela. Sus principales líneas de investigación son la Aplicación de nuevas metodologías en Educación Primaria e Infantil. Además participa como investigadora en el Departamento de Química Analítica 
y Alimentaria en la Universidad de Vigo en el proyecto del Ministerio de Economía y Competitividad, convocatoria de proyectos I+D del programa estatal de fomento de la investigación científica y técnica de excelencia: "Detección on-site de iones, complejos metálicos y nanopartículas mediante estrategias nanoanalíticas basadas en grafeno y puntos cuánticos de carbono”.

$\triangle$ sandra.fragueirobarreiro@ceu.es

María Pousa Ruibal. Graduada en Educación Infantil por la Escuela Universitaria CEU de Magisterio de Vigo (Universidad de Vigo, 2018). Actualmente sus principales líneas de investigación se centran en el estudio y puesta en práctica de metodologías innovadoras en la etapa educativa de Educación Infantil.

$\square$ mariapouru@gmail.com 